\title{
Error Bound of the Stochastic Linear Complementarity ${ }^{*}$
}

\author{
Shenghua Jiao', Qingjun Ren ${ }^{2}$ \\ ${ }^{1)}$ School of Logistics, ${ }^{2)}$ School of Science, LinYi University, Linyi, Shandong Province, China
}

\begin{abstract}
In this paper, the error bound estimation for linear complementarity problem are extended to the stochastic linear complement problem (SLCP). Some of its new reformulations are given firstly, and then we establish its error bound estimation and its solution structure.
\end{abstract}

Index Terms -SLCP, new reformulations, error bound, solution structure.

\section{Introduction}

$$
\text { Let } F(x, \omega):=M(\omega) x+p(\omega), G(x, \omega):=N(\omega) x+q(\omega)
$$

Where $M(\omega), N(\omega) \in R^{n \times n}$ are stochastic matrices; $p(\omega)$,

$q(\omega) \in R^{n}$ are random variables. If $M(\omega), N(\omega)$ are only numerical value, then we get the Linear Complementarity Problem(LCP). The SLCP is an extension case of the LCP which has received much attention, some efficient solution methods are given for it [1-3], begin to play significant role in economics, operation research, and stochastic analysis, etc.

If $R^{n}$ is finite dimension, then the SLCP is equivalent to $\bar{F}(x):=\bar{M}(\omega) x+\bar{p}(\omega), \bar{G}(x, \omega):=\bar{N}(\omega) x+\bar{q}(\omega)[4]$, where $\bar{M}=\bar{M}(\omega)=\sum_{i=1}^{\infty} p_{i} M\left(\omega_{i}\right), \bar{P}=\bar{p}(\omega)=\sum_{i=1}^{\infty} p_{i} q\left(\omega_{i}\right)$.

Our goal is to find a vector $x^{*} \in R^{n}$ such that

$$
\bar{F}\left(x^{*}\right) \in K, \bar{G}\left(x^{*}\right) \in K^{0}, \bar{F}\left(x^{*}\right)^{T} \bar{G}\left(x^{*}\right)=0
$$

Where $K$ is a polyhedral cone in $R^{n}$, and $K^{0}$ is its dual cone. There exist matrices $A, B \in R^{n \times n}$, such that

$$
\begin{gathered}
K=\left\{v \in R^{n} \mid A v \geq 0, B v=0\right\} \\
K^{0}=\left\{u \in R^{n} \mid A^{T} \lambda_{1}+B^{T} \lambda_{2}, \lambda_{1} \geq 0, \lambda_{1}, \lambda_{2} \in R^{n}\right\}
\end{gathered}
$$

We denote its solution set by $X^{*}$, which is assumed to be nonempty throughout this paper. Obviously, the SLCP is an extension of the LCP, and this motivates us to consider its error bound estimation. So we will establish a new error bound estimation based on some new reformulations of the problem and also discuss its solution structure. Compared with the error bound given before, the error bound given here is more easily.

\section{Equivalent reformulation}

In this section, we will establish an equivalent reformulation of the SLCP. First, we need the follow assumptions.

Assumption: For matrices $A, B, M, N$ involved in (1.1), it holds that

(1) The matrix $\boldsymbol{M}^{T} \boldsymbol{N}$ is positive semi-definite;

(2) The matrix $\left(\boldsymbol{A}^{T}, \boldsymbol{B}^{T}\right)$ has full-column rank.

For the LCP, referring the reader to the argument in [5], and it is easy to deduce the following conclusion in the SLCP.

A point $x^{*} \in R^{n}$ is a solution of the stochastic linear complement if and only if there exist $\lambda_{1}, \lambda_{2} \in R^{n}$, such that

$$
\left\{\begin{array}{l}
A\left(\bar{M} x^{*}+\bar{p}\right) \geq 0 \\
B\left(\bar{M} x^{*}+\bar{p}\right)=0 \\
\lambda_{1} \geq 0 \\
\left(\bar{M} x^{*}+\bar{p}\right)^{T}\left(\bar{N} x^{*}+\bar{q}\right)=0 \\
\bar{N} x^{*}+\bar{q}=A^{T} \lambda_{1}+B \lambda_{2}
\end{array}\right.
$$

Denote $Q=\left(\begin{array}{l}A \\ B\end{array}\right)$, then $Q$ has full-row rank, and thus the 2n-order matrix $Q Q^{T}$ is non-singular. So we have the following conclusion.

Theorem 2.1 Suppose Assumption (2) holds. For any $x \in R^{n}$, the following statements are equivalent.

1. There exist $\lambda_{1} \in R_{+}^{s}, \lambda_{2} \in R^{t}$, such that

$$
\bar{N}_{x}+\bar{q}=A^{T} \lambda_{1}+B^{T} \lambda_{2}
$$

$$
\text { 2. }\left\{\begin{array}{l}
\left(I_{s}, 0_{s \times t}\right)\left(Q Q^{T}\right)^{-1} Q(\bar{N}(x+\bar{q}) \geq 0 \\
{\left[Q^{T}\left(Q Q^{T}\right)^{-1} Q-I_{m}\right](\bar{N} x+\bar{q})=0}
\end{array}\right.
$$

$$
\begin{array}{r}
\text { Proof Set } X_{1} \triangleq\left\{x \in R^{n} \mid \bar{N} x+\bar{q}=A^{T} \lambda_{1}+B^{T} \lambda_{2}\right\} \\
X_{2} \triangleq\left\{x \in R^{n} \mid \begin{array}{l}
\left(I_{s}, 0_{s \times t}\right)\left(Q Q^{T}\right)^{-1} Q(\bar{N} x+\bar{q}) \geq 0 \\
{\left[Q^{T}\left(Q Q^{T}\right) Q-I_{m}\right](\bar{N} x+\bar{q})=0}
\end{array}\right\}
\end{array}
$$

For any $x \in X_{1}$, there exists $\lambda_{1} \in R_{+}^{n}, \lambda_{2} \in R^{n}$, such that

$$
\bar{N} x+\bar{q}=Q^{T}\left(\begin{array}{l}
\lambda_{1} \\
\lambda_{2}
\end{array}\right) \text {. }
$$

\footnotetext{
* This work was supported by Shandong Province Science and Technology Development Projects (2013GGA13034), the Chinese Society of Logistics and the China Federation of Logistics and Purchasing Project (2015CSLKT3-199), the Logistics Teaching and Research Reformation Projects for Chinese Universities (JZW2014048, JZW2014049). ${ }^{2}$ Ren is the corresponding author (e-mail: shua2020@163.com.cn, phone:13645496022)。
} 
Therefore $\left(Q Q^{T}\right)^{-1} Q(\bar{N} x+\bar{q})=\left(\begin{array}{l}\lambda_{1} \\ \lambda_{2}\end{array}\right)$

We have

$$
\left[Q^{T}\left(Q Q^{T}\right)^{-1}-I_{m}\right](\bar{N} x+\bar{q})=0
$$

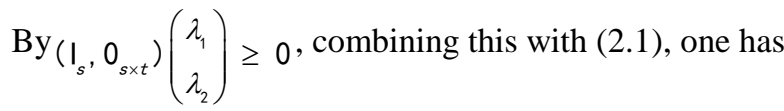

$$
\left(I_{s}, 0_{s \times t}\right)\left(Q Q^{T}\right) Q(\bar{N} x+\bar{q}) \geq 0
$$

Along with (2.3), yields that $x \in X_{2}$.

On the other hand, for any $x \in X_{2}$, let

$$
\begin{aligned}
& \lambda_{1}=\left(I_{s}, O_{s \times t}\right)\left(Q Q^{T}\right)^{-1} Q(\bar{N} x+\bar{q}), \\
& \lambda_{2}=\left(0_{s \times t}, I_{s}\right)\left(Q Q^{T}\right)^{-1} Q(\bar{N} x+\bar{q})
\end{aligned}
$$

Then $\lambda_{1} \in R_{+}^{s}, \lambda_{2} \in R_{+}^{t}$. By(2.3)得

$$
\begin{aligned}
& \bar{N} x+\bar{q}=Q^{T}\left[\left(Q Q^{T}\right)^{-} Q(\bar{N} x+\bar{q})\right] \\
& =\left(A^{T}, B^{T}\right)\left(\begin{array}{ll}
I_{s} & 0 \\
0 & I_{t}
\end{array}\right)\left(Q Q^{T}\right)^{-1} Q(\bar{N} x+\bar{q})=A^{T} \lambda_{1}+B^{T} \lambda_{2} .
\end{aligned}
$$

In fact, Theorem 2.1 transforms the third inequality and the last equality in (1.2) into a new system of inequalities in which neither parameter $\lambda_{1}$ or $\lambda_{2}$ is not involved. In this sense, the SLCP can be reformulated as the following system.

$$
\left\{\begin{array}{l}
A(\bar{M} x+\bar{p}) \geq 0 \\
B(\bar{M} x+\bar{p})=0 \\
\left(A\left(\bar{M}_{x}+\bar{p}\right)\right)^{T}\left(I_{s}, 0_{s \times t}\right)\left(Q Q^{T}\right)^{-1} Q\left(\bar{N}_{x}+\bar{q}\right)=0 \\
\left(I_{s}, 0_{s \times t}\right)\left(Q Q^{T}\right)^{-1} Q\left(\bar{N}_{x}+\bar{q}\right) \geq 0 \\
\left(Q^{T}\left(Q Q^{T}\right)^{-1} Q-I_{m}\right)\left(\bar{N}_{x}+\bar{q}\right)=0
\end{array}\right.
$$

By (5),Question (1) From (2.4), problem (1.1) can be equivalently reformulated as the following constrained optimization problem.

$$
\min H(x)=(\bar{M} x+\bar{p})^{T}(\bar{N} x+\bar{q}) \quad \text { s. } t . x \in X \quad \text { (2.5) }
$$

where

$$
x=\left\{x \in R^{n}\left\{\begin{array}{l}
A\left(\bar{M}_{x}+\bar{p}\right) \geqslant 0,\left(I_{s}, O_{s \times s}\right)\left(Q Q^{T}\right)^{-1} Q\left(\bar{N}_{x}+\bar{q}\right) \geqslant 0 \\
\left.B(\bar{M} x+\bar{p})=0,\left(Q^{\top}\left(Q Q^{\top}\right)\right)^{-1}-I_{m}\right)\left(\bar{N}_{x}+\bar{q}\right)=0
\end{array}\right\}\right.
$$

In the sense, that $X^{*}$ is a solution of (1.1) if and only if it is a global optimal solution of (2.5).

From Assumption (1), the Hessian-matrix $\bar{M}^{T} \bar{N}+\bar{N}^{T} \bar{M}$ of $H(x)$ is positive semi-definite, so $H(x)$ is a convex function, the feasible set is a polyhedral. Thus, by the related optimal theory, we know that the stationary set of (2.5) coincides with its solution set which also coincides with $x \in X^{*}$, such that

$$
\left(\mathrm{x}-\mathrm{x}^{*}\right)^{T}\left(M \mathbf{x}^{*}+q\right) \geq 0, \forall x \in \mathrm{X}
$$

Where $M=\bar{M}^{T} \bar{N}+\bar{N}^{T} \bar{M}, q=\bar{M}^{T} \bar{q}+\bar{N}^{T} \bar{p}$. From (2.6), we can detect the structure of the solution set.

Theorem 2.2 Suppose that Assumption (1) and (2) holds and $x_{0}$ is a solution of (1.1), then $\mathbf{x}^{*}=\left\{x \in X \mid M\left(\mathbf{x}-x_{0}\right)=0, q\left(\mathbf{x}-x_{0}\right)=0\right\}$.

Proof Set $W=\left\{w \in X \mid M\left(\mathbf{x}-x_{0}\right)=0, q\left(\mathbf{x}-x_{0}\right)=0\right\}$, $\forall x \in \mathrm{X}^{*}$, since $x, x_{0} \in X$, from (2.6), one has

$$
\begin{aligned}
& \left(x-x_{0}\right)^{T}\left(M x_{0}+q\right) \geq 0 ; \\
& \left(x_{0}-x\right)^{T}(M x+q)=0 .
\end{aligned}
$$

Adding these two inequalities yields

$$
\left(x-x_{0}\right)^{T} M\left(x-x_{0}\right) \leq 0,
$$

Since $M$ is symmetric and positive semi-definite by (1), so

$$
M\left(x-x_{0}\right)=0 .
$$

From (2.7), one has $\left(\tilde{x}-x_{0}\right)^{\top}\left(\tilde{M} x_{0}+\bar{q}\right)=\left(\tilde{x}-x_{0}\right)^{\top} \tilde{M}\left(x_{0}-\tilde{x}\right)+\left(x_{0}-\tilde{x}\right)^{\top}(\tilde{M} \tilde{x}+\bar{q}) \geq 0 C$ ombining this with (2.8) yields

$$
\left(\tilde{\mathbf{x}}-x_{0}\right)^{T}\left(M \mathbf{x}_{0}+\bar{q}\right)=0,
$$

Using (2.10), we have

$$
q^{T}\left(x-x_{0}\right)=\left(x_{0}-x\right)^{T} M x_{0}=0
$$

By (2.10) and (2.12), we get $x \in W$.

On the other hand, for any $\omega \in W$ and $x \in X$, since

$$
M\left(\omega-x_{0}\right)=0, q^{T}\left(\omega-x_{0}\right)=0
$$

We have

$(x-\omega)^{T}(M \omega+q)$

$=\left[\left(x-x_{0}\right)+\left(x_{0}-\omega\right)\right]^{T}\left[M\left(\omega-x_{0}\right)+\left(M x_{0}+q\right)\right]$

$=\left[\left(x-x_{0}\right)+\left(x_{0}-\omega\right)\right]^{T}\left(M x_{0}+q\right)$

$=\left(x-x_{0}\right)^{T}\left(M x_{0}+q\right) \geq 0$.

Where the inequality follows from that $x_{0}$ is a solution of the SLCP, thus $\omega \in X^{*}$.

From Theorem 2.2, it is easy to get the following conclusion.

Coraollary2.1 Suppose (1) and (2) hold, and $x_{0}$ is a solution of (1.1). Then

$$
X^{*}=\left\{x \in X \mid M\left(x-x_{0}\right)=0,\left(M x_{0}+q\right)^{T}\left(x-x_{0}\right) \leq 0\right\}
$$

In the following, we would give the definition which plays a crucial role in discussing the structure of the solution set for the SLCP which is developed based on complementarity conditions in (2.4).

Definition 2.1 A solution $x$ of (1) is said to be nondegenerate if it satisfies 


$$
A(\bar{M} x+\bar{p})+\left(I_{n}, 0_{n \times n}\right)\left(Q Q^{T}\right)^{-1} Q(\bar{N} x+\bar{q})>0 .
$$

Theorem 2.3 Suppose that (1) and 2) holds, and the SLCP has a non-degenerate solution. Then for any solution $x_{0}$ of (1.1), it holds that

$$
x^{*}=\left\{x \in X \mid(\bar{M} x+\bar{p})^{T}\left(\bar{N} x_{0}+\bar{q}\right)(\bar{N} x+\bar{q})^{T}\left(\bar{M} x_{0}+\bar{p}\right)\right.
$$

Proof For any $x \in X$, by the second equality in*, we have $\left(x-x_{0}\right)^{T}\left(\bar{M}^{T} \bar{N}+\bar{N}^{T} \bar{M}\right)\left(x-x_{0}\right)$

$=\left[(\bar{M} x+\bar{p})-\left(\bar{M} x_{0}+\bar{p}\right)\right]^{\top}\left[(\bar{N} x+\bar{q})-\left(\bar{N} x_{0}+\bar{q}\right)\right](2.14)$

$+\left[(\bar{N} x+\bar{q})-\left(\bar{N} x_{0}+\bar{q}\right)\right]^{\top}\left[\left(\bar{M}_{x}+\bar{p}\right)-\left(\bar{M}_{0}+\bar{p}\right)\right]$

$=2(\bar{M} x+\bar{p})^{T}(\bar{N} x+\bar{q})-2(\bar{M} x+\bar{p})^{T}\left(\bar{N} x_{0}+\bar{q}\right)-2\left(\bar{M} x_{0}+\bar{p}\right)^{T}(\bar{N} x+\bar{q})$

To prove the assertion, we need that the solution set $X^{*}$ and $W$ are equivalent, where

$W=\left\{x \in X \mid(\bar{M} x+\bar{p})^{T}\left(\bar{N} x_{0}+\bar{q}\right)+(\bar{N} x+\bar{q})^{T}\left(\bar{M} x_{0}+\bar{p}\right)=0\right\}$

For any $x \in X^{*}$, by Theorem 2.2, one has $(\bar{M} x+\bar{p})^{T}(\bar{N} x+\bar{q})=0,\left(\bar{M}^{T} \bar{N}+\bar{N}^{T} \bar{M}\right)^{T}\left(x-x_{0}\right)=0$

Combining this with $(2.14)$,we have

$$
(\bar{M} x+\bar{p})^{T}\left(\bar{N} x_{0}+\bar{q}\right)+\left(\bar{M}^{T} x_{0}+\bar{p}\right)^{T}(\bar{N} x+\bar{q})=0 .
$$

This implies that $X^{*} \subseteq W$.

On the other hand, if $\bar{x}$ is a non-degenerate solution of the SLCP. From the theory just proved, we have

$$
\left(\bar{M} x_{0}+\bar{p}\right)^{T}(\bar{N} \bar{x}+\bar{q})+\left(\bar{N} x_{0}+\bar{q}\right)^{T}(\bar{M} x+\bar{p})=0
$$

Now for any $X \in W$, by Theorem 2.2 the second equality in (2.4) and (2.15)

$$
\begin{aligned}
& 0=\left(x-x_{0}\right)^{T}\left(\bar{M}^{T} \bar{N}+\bar{N}^{T} \bar{M}\right)\left(x-x_{0}\right) \\
& =(\bar{M} x+\bar{p})^{T}(\bar{N} x+\bar{q})+(\bar{N} x+\bar{q})^{T}(\bar{M} x+\bar{p}) \\
& -(\bar{M} x+\bar{p})^{T}\left(\bar{N} x_{0}+\bar{q}\right)+(\bar{N} x+\bar{q})^{T}\left(\bar{M} x_{0}+\bar{p}\right) .
\end{aligned}
$$

We have

$$
\begin{aligned}
& (\bar{M} x+\bar{p})^{T}(\bar{N} \bar{x}+\bar{q})^{T}+(\bar{N} x+\bar{q})^{T}\left(\bar{M}_{x}+\bar{p}\right)=0 \\
& (\bar{M} x+\bar{p})^{T}(\bar{N} x+\bar{q})^{T}+(\bar{N} x+\bar{q})^{T}\left(\bar{M}_{x}+\bar{p}\right)=0 .
\end{aligned}
$$

However, by the third equality in (2.4), one has

$$
\begin{aligned}
& (\bar{M} \mathrm{x}+\bar{p})(\bar{N} \overline{\mathrm{x}}+\bar{q})=(\bar{M} \mathrm{x}+\bar{p})^{T}\left[\theta^{T}\left(\theta \theta^{T}\right)^{-1} \theta(\bar{N} \overline{\mathrm{x}}+\bar{q})\right. \\
& \left.=(\bar{M} \times x+\bar{p})^{T}(\bar{N} \bar{x}+\bar{q})+(\bar{N} \mathrm{x}+\bar{q})^{T}(\bar{M} \overline{\mathrm{x}}+\bar{p})\right] \\
& =\left[\left(\begin{array}{l}
A \\
B
\end{array}\right)(\bar{M} \mathrm{x}+\bar{p})\right]^{T}\left(\begin{array}{cc}
I_{s} & 0 \\
0 & I_{s \times t}
\end{array}\right)\left(\theta \theta^{T}\right)^{-1} \theta(\bar{N} \overline{\mathrm{x}}+\bar{q}) \\
& =(A(\bar{M} \mathrm{x}+\bar{p}))^{T}\left[\left(I_{s}, 0_{s \times t}\right)\left(\theta \theta^{T}\right)^{-1} \theta(\bar{N} \bar{x}+\bar{q})\right], \\
& (\bar{N} \mathrm{x}+\bar{q})(\bar{M} \overline{\mathrm{x}}+\bar{p})=\left[\theta^{T}\left(\theta \theta^{T}\right)^{-1} \theta(\bar{N} \mathrm{x}+\bar{q})^{T}(\bar{M} \overline{\mathrm{x}}+\bar{p})\right] \\
& =\left[\left(\theta \theta^{T}\right)^{-1} \theta(\bar{N} \mathrm{x}+\bar{q})\right]^{T}[\theta(\bar{M} \overline{\mathrm{x}}+\bar{p})] \\
& =\left[\left(\begin{array}{cc}
I_{s} & 0 \\
0 & I_{s \times t}
\end{array}\right)\left(\theta \theta^{T}\right)^{-1} \theta(\bar{N} \mathrm{x}+\bar{q})\right]\left[\left(\begin{array}{l}
A \\
B
\end{array}\right)(\bar{M} \overline{\mathrm{x}}+\bar{p})\right] \\
& =\left[\left(I_{s}, 0_{s \times t}\right)\left(\theta \theta^{T}\right)^{-1} \theta(\bar{N} \mathrm{x}+\bar{q})\right]^{T}[A(\bar{M} \overline{\mathrm{x}}+\bar{p})], \\
& {[A(\bar{M} x+\bar{p})]^{T}\left[\left(I_{s}, 0_{s \times t}\right)\left(Q Q^{T}\right)^{-1} Q(\bar{N} \bar{x}+\bar{q})\right]=0}
\end{aligned}
$$

Combining this with (2.11), we can get

$$
[A(\bar{M} x+\bar{p})]^{T}\left[\left(I_{s}, 0_{s \times t}\right)\left(\theta \theta^{T}\right)^{-1} \theta(\bar{N} x+\bar{q})\right]=0 .
$$

And from (2.4), one has

$$
\begin{gathered}
(\bar{M} x+\bar{p})^{T}(\bar{N} \bar{x}+\bar{q})^{T}+(\bar{N} x+\bar{q})^{T}(\bar{M} \bar{x}+\bar{p})=0 \\
\left(\bar{M}_{x}+\bar{p}\right)^{T}(\bar{N} x+\bar{q})=0
\end{gathered}
$$

So we have $x \in X *$.

From Theorem 2.3 and (2.4), it is easy to prove the following conclusion.

Corollary 2.2 If the conditions of Theorem 2.3 hold. Then the structure of solution set for the stochastic linear complement has the following representation.

$$
X^{*}=\left\{x \in X \mid(\bar{M} x+\bar{p})^{T}\left(\bar{N} x_{0}+\bar{q}\right)+(\bar{N} x+\bar{q})^{T}\left(\bar{M} x_{0}+\bar{p}\right) \leq 0\right\}
$$

\section{The error bound for the stochastic linear complement}

In this section, we present the global error bound for the SLCP based on the structure of solution set obtained in previous section under Assumption (1) and (2). Now, we mainly state our main results and firstly give the needed lemma.

Lemma 3.1 Suppose $B_{1} x+b_{1} \geqslant 0, D_{1} x+d_{1}=0$, 则

$$
\|x\| \geq\left[\left\|\left(-b_{1}\right)_{+}\right\|+\left\|d_{1}\right\|\right] /\left[\left\|B_{1}\right\|+\left\|D_{1}\right\|\right]
$$

Proof $\mathrm{By} B_{1} x+b_{1} \geqslant 0$, for any $i$, we can easily get $\left(B_{1} x\right)_{i}^{2} \geq\left[\left(-b_{1}\right)_{+}\right]_{i}^{2}$, this means $\left\|B_{1} x\right\| \geq\left\|\left(-b_{1}\right)_{+}\right\| \geqslant 0$.

Combining with $D_{1} x+d_{1}=0$, we obtain the desired result.

In this following, we mainly quote some known results from [6] on the error bound for a polyhedral cone which will be used in the sequel.

Lemma 3.2 Given polyhedral cone

$$
P=\left\{x \in R^{n} \mid D_{3} x=d_{3}, B_{3} x \leq b_{3}\right\}
$$

Where $D_{3} \in R^{/ \times n}, B_{3} \in R^{m \times n}, d_{3} \in R^{\prime}, b_{3} \in R^{m}$. For any

$x \in R^{n}$, then there exists a constant $c_{1}>0$

Such that

$$
\operatorname{dist}(x, P) \leq C_{1}\left[\left\|D_{3} x-d_{3}\right\|+\| \max \left\{0, B_{3} x-b_{3} \|\right. \text {. }\right.
$$

From Corollary 2.2 and Lemma 3.1-3.2, we have the global error bound for the SLCP.

Theorem 3.1 Suppose that Assumption (1) and (2) holds. Then there exists a constant $\gamma>0$, for any $x \in R^{n}$, we can find a vector $x^{*} \in R^{n}$ such that

$$
\begin{aligned}
& \left\|x-x^{*}\right\| \leq \gamma\left\{m(x)+m(x)^{1 / 2}\right\} \\
& \left\|x-x^{*}\right\| /\left\|x^{*}\right\| \leq \rho\left\{m(x)+m(x)^{1 / 2}\right\}
\end{aligned}
$$

where 
$m(x)=\|A(\bar{M} x+\bar{p})-\|+\left\|(I, 0)\left(\theta \theta^{T}\right)^{-1} \theta(\bar{N} x+\bar{q}).\right\|+\|B(\bar{M} x+\bar{p})\|$

$+\|\bar{\theta}(\bar{N} x+\bar{q})\|+\left[(\bar{M} x+\bar{p})^{T}(\bar{N} x+\bar{q})\right]_{+}$,

$\Gamma=\left\|\left(\begin{array}{l}-A \bar{p} \\ (I, 0)\left(\theta \theta^{T}\right)^{-1} \theta \bar{q}\end{array}\right)_{+}\right\| \neq 0, \lambda=\left\|\left(\begin{array}{l}A \bar{M} \\ (I, 0)\left(\theta \theta^{T}\right)^{-1} \theta \bar{N}\end{array}\right)\right\|$,

$\rho=\gamma \Gamma^{-1} \lambda$, and $\bar{\theta}=\theta^{T}\left(\theta \theta^{T}\right)^{-1} \theta-I_{m}$

Proof From (2.8) and Lemma 3.2, for any $x \in R^{n}$, there has a constant $\delta_{1}>0$ such that

$\left\|x-x^{*}\right\| \leq \delta_{1}\left\{\left\|[A(\bar{M} x+\bar{p})]_{-}\right\|+\|(I, 0)\left(\theta \theta^{T}\right)^{-1} \theta(\bar{N} x+\bar{q})\right] . \|$

$+\|B(\bar{M} x+\bar{p})\|+)+\left\|\left(\bar{M}^{T} \bar{N}^{T} \bar{N}^{T} \bar{M}\right)\left(x-x_{0}\right)\right\|$

$\left.+\left\|\left[\left(M x_{0}+q\right)^{T}\left(x-x_{0}\right)\right]_{+}\right\|\right\}$,

where $x_{0} \in X^{*}$. By $\left.H(x):=\overline{(M x}+\bar{p}\right)^{T}(\bar{N} x+\bar{q})$ is convex

and $H\left(x_{0}\right)=0$, we have

$$
\begin{gathered}
H(x)-H\left(x_{0}\right) \geq\left(M x_{0}+q\right)^{T}\left(x-x_{0}\right) \text { i.e., } \\
{\left[\left(M x_{0}+q\right)^{T}\left(x-x_{0}\right)\right]_{+} \leq\left[\left(M x_{0}+q\right)^{T}(\bar{N}(x)+\bar{q})\right]_{+}}
\end{gathered}
$$

For any $x \in R^{n}$ there exists a constant $\delta_{2}$, such that

$$
\left\|\left(\bar{M}^{T} \bar{N}+\bar{N}^{T} \bar{M}\right)\left(x-x_{0}\right)\right\|^{2} \leq \delta_{2}\left(x-x_{0}\right)^{T}\left(\bar{M}^{T} \bar{N}+\bar{N}^{T} \bar{M}\right)\left(x-x_{0}\right)
$$

$=2 \delta_{2}\left\{(\bar{M} x+\bar{P})^{T}(\bar{N} x+\bar{q})-\left(\bar{M} x_{0}+\bar{P}\right)^{T}\left(\bar{N} x_{0}+\bar{q}\right)\right.$

$\left.-\left[\left(\bar{M}^{T} \bar{N}+\bar{N}^{T} \bar{M}\right) x_{0}+\left(\bar{M}^{T} \bar{q}+\bar{N}^{T} \bar{P}\right)\right]^{T}\left(x-x_{0}\right)\right\}$

$\leq 2 \delta_{2}\left\{\left[(\bar{M} x+\bar{P})^{T}(\bar{N} x+\bar{q})\right]_{+}\right.$

$\left.\left.+\left[\left(\bar{M}^{T} \bar{N}+\bar{N}^{T} \bar{M}\right)_{x_{0}}+\left(\bar{M}^{T} q+\bar{N}^{T} \bar{P}\right)\right]^{T}\left(x-x_{0}\right)\right]\right\}_{-}$

$\leq 2 \delta_{2}\left[(\bar{M} x+\bar{p})^{T}(\bar{N} x+\bar{q})\right]_{+}$

$+2 \delta_{1} \delta_{2}\left(\left\|[A(\bar{M} x+\bar{p})]_{-}\right\|+\left\|\left[(/, 0)\left(Q Q^{T}\right)^{-1} Q(\bar{N} x+\bar{q})\right]_{-}\right\|\right.$

$+\|\bar{Q}(\bar{N} x+\bar{q})\|+\|B(\bar{M} x+\bar{p})\|)$,

Where the first inequality is by Assumption (1), the equality is by the Taylor expansion equation of $H(x)$ at $x_{0}$, the second inequality follows from the fact $a+b \leq a_{+}+b_{+}, \forall a, b$.the last inequality is by (2.2), and $x_{0} \in X^{*}$. From (3.4)-(3.6), we have that (3.2) holds. Combining (3.2) with Lemma 4.1, we deduce that (3.3) holds.

This bound is an extension of Theorem 2.7 in [7]. Similar to the derivation of Theorem 3.1, we can also obtain an error bound for (1.1) under the condition of Theorem 2.3.

Theorem 3.2 Suppose that the condition of Theorem 2.3 holds. Then there exists a constant $\rho_{1}>0$, for any $x \in R^{n}$, we can find a vector $x^{*} \in X^{*}$, such that

$$
\left\|x-x^{*}\right\| \leq \rho_{1} m(x)
$$

$$
\left\|x-x^{*}\right\| /\left\|x^{*}\right\| \leq \rho_{2} m(x) .
$$

Where $m(x), \theta \neq 0, \lambda$ are defined in Theorem 3.1 ,$\rho_{2}=\rho_{1} \theta^{-1} \lambda$.

Proof For any $x \in R^{n}$, there exists a constant $\delta_{3}>0$, such that

$$
\begin{aligned}
& \left\|x-x^{*}\right\| \leq \delta_{3}\left\{\left\|A(\bar{M} x+p)_{-}\right\|+\|(I, 0)\left(Q Q^{T}\right)^{-1} Q(\bar{N} x+\bar{q})\right]_{-} \| \\
& +\|B(\bar{M} x+\bar{p})\|+\left\|\bar{Q}\left(\bar{N}_{x}+\bar{q}\right)\right\| \\
& \left.+\left\|\left[(\bar{M} x+p)^{T}\left(\bar{N}_{x_{0}}+q\right)+(\bar{N} x+q)^{T}\left(\bar{M}_{x_{0}}+p\right)\right]_{+}\right\|\right\} \\
& \leq \delta_{3}\left\{\left\|A\left(\bar{M}_{v}+\bar{p}\right)_{-}\right\|+\|(/, 0)\left(Q Q^{T}\right)^{-1} Q(\bar{N} x+\bar{q})\right]_{-} \| \\
& +\|B(\bar{M} x+\bar{p})\|+\left\|\bar{Q}\left(\bar{N}_{x}+\bar{q}\right)\right\| \\
& \left.+\left\|\left[(\bar{M} x+\bar{p})^{T}\left(\bar{N}_{x}+\bar{q}\right)\right]_{+}\right\|\right\},
\end{aligned}
$$

Where $x_{0} \in X^{*}$, the first inequality is by (2.16) and Lemma 3.2, and the second inequality is by (2.16) and Assumption (1). Combining (3.7) with Lemma 3.1, we obtain (3.8).

\section{Acknowledgment}

The authors would like to thanks the referees for helpful suggestions and comments especial from Professor Hong chun Sun of LinYi University. This work was supported by some funds listed under the home page.

\section{References}

[1] Ling H, Chen X J and Fukushima M, "New restricted NCI functions and their applications to stochastic NCP and stochastic NPEC," Optimization, 2007, vol.56, pp.641-653.

[2] Ling $\mathrm{H}$ and Fukushima M, "New reformulations for stochastic nonlinear complementarity problems", Optim Methods Soft, 2006,vol.21, pp.551564.

[3] Zhou G L and Caccetral, "Feasible semi-smooth Newton method for a class of stochastic linear complementarity," Optim Theory App1., 2008, vol 139, pp.379-392.

[4] Zhang and GAO, "Superlinner /quadratic one-step smoothing Newton method for PO-NCP without strict complementarity," Mathematical Methods of Operation Reserch,2002, vol.56,pp.231-241.

[5] Y.J. Wang, F.M. Ma and J.Z. Zhang, "A nonsmooth L-M method for solving the generalized nonlinear complementarity problem over a polyhedral cone", Appl. Math. Optim.2005,vol.52(1).pp.73-92.

[6] A.J. Ho_man, "On the approximate solutions of linear inequalities", Res. National Bureau of Standards, 1952, vol.49.pp 263-265.

[7] P. Tseng, "Growth behavior of a class of merit function for the nonlinear complementarity problem," Optim. Theory Appl., 1996, vol.89.pp.1737. 